\title{
The rabbit and cat in the delayed alternation problem'
}

Seven rabbits and seven cats were trained in a single alternation sequential task with minimum delay. Delay was then introduced between trials through 10-sec., 15-sec., 20-sec. and 30-sec. delay periods. Five rabbits and six cats attained criterion performance at all delay levels. On the delayed alternation trials, in contrast to alternation with minimum delay, cats learned the task significantly more rapidly than did rabbits. This result was in the anticipated direction.

The delayed response problem, originated by Hunter in 1913 "remains the one behavioral test most sensitive to the widest range of experimental treatments" (Fletcher, 1965).

Hunter (1917) claimed that tasks calling for delay of response differentiated between animals in different classes, and Fletcher (1965) concludes that "recent experiments...have verified the general correlation between phyletic status and performance on the delayed response problem." Fletcher, however, points to wide variations in delay that can be obtained with different techniques and Warren (1965) on these grounds and in the light of wide individual differences evident within species, disputed that it may be claimed that primates are superior to subprimates on this task.

Closely associated with delayed response is delayed alternation. It has been demonstrated that severe decrement in delayed response performance follows prefrontal cortical ablation (e.g., Pribram, Mishkin, Rosvold, \& Kaplan, 1952; Miles \& Blomquist, 1960) and a similar result has been observed for delayed alternation (Jacobsen \& Nissen, 1937; Mishkin \& Pribram, 1956). Jacobsen \& Nissen (1937) point out that, in the former task, the experimenter presents the cue before each delay while, in the latter, the cue is given by "external and internal stimuli conditioned by its own overt response to one of the containers on the just preceding trial."

The rabbit and cat differ in cephalic index of brainbody ratio, the cat having the higher index (Von Bonin, 1937) and carnivores have been shown to be superior to rodents in a number of learning and problem-solving tasks (Warden, 1951). It was therefore considered worthwhile to test representatives of these two species on a form of the delayed alternation problem, to see whether this problem would differentiate between them in favor of the cat.

\section{Method}

Subjects. The Ss were four male and three female rabbits and seven male cats from the group of 10 rabbits and eight cats already described (Livesey, 1965). At the commencement of testing the rabbits were 2-3 yr. of age and the cats $2-1 / 2$ to $3-1 / 2 \mathrm{yr}$. old. All Ss had completed the Hebb-Williams Closed Field Test (Livesey, 1966), the Hebb-Williams Elevated Pathway Test, a discrimination learning task and the Double Alternation Problem (Livesey, 1965). Housing and living conditions for these Ss have already been described (Livesey, 1965).

Apparatus. The WGTA described in Livesey (1964) was used for testing of all Ss.

Procedure. 1. Adaptation to apparatus. A Klüver tray with one centrally placed food well was used (a) with no covering to food well; $S$ was required to complete 25 responses (removing food from well each time presented) within $15 \mathrm{~min}$; (b) food well covered by block, with food always present; $S$ required to complete $\mathbf{5 0}$ responses within $30 \mathrm{~min}$. for four consecutive days.

2. Forced alternation training. A tray with two food wells as for double alternation was used. One compartment was uncovered and empty, the other was covered and contained food. The food was alternated from one compartment to the other on each trial. The $S$ was required to complete 50 trials a day for four days with an average time of not more than $10 \%$ above that which it achieved in 1-b above.

3. Alternation training minimum delay. Both food wells were covered with identical blocks. The food was alternated after each correct response. The $\mathrm{S}$ completed 50 trials per day. Criterion of successful performance was an average of $80 \%$ correct responses over four consecutive days, i.e., 160 correct responses out of 200. Delay between trials was approximately $5 \mathrm{sec}$.

4. Delayed alternation. With procedure as for 3 , delay was introduced between trials. Delays were successively $10 \mathrm{sec} ., 15 \mathrm{sec} ., 20 \mathrm{sec}$., and $30 \mathrm{sec}$. On attaining criterion performance on one delay level, $\mathrm{S}$ was then shifted to the next longest delay.

5. Definition of a trial. The opaque screen was lowered, separating the $\mathrm{S}$ from the food tray, and the one-way screen was raised. This completed one trial and began the next. While the opaque screen was lowered, the food was placed in the appropriate container; the one-way screen was then lowered. The opaque screen remained lowered for the period of the delay, this being determined as the time between the lowering of this screen and the subsequent raising of it. The screen was raised and the $\mathrm{S}$ responded by displacing a block from a food well and, if correct, removing the food. The screen was lowered.

6. Method of alternation. The correct response was to the food well not responded to on the previous trial, whether that trial was correct or not. 
Table 1. Alternation with minimum delay, number of days to criterion* for cats and rabbits

\begin{tabular}{lcccccccc} 
Animal No. & 1 & 2 & 3 & 4 & 5 & 6 & 7 & $\bar{X}$ \\
\hline Species & & & & & & & & \\
Cats & (a) & 27 & 21 & 11 & 15 & 30 & 19 & 20.15 \\
Rabbits & 18 & 21 & 24 & 26 & (b) & 14 & 22 & 21.00 \\
\hline
\end{tabular}

* This number includes the 4 days during which criterion performance was being achieved.

(a) Failed to reach criterion

(b) Died before reaching criterion

Thus $S$ must always alternate to receive reward. Results

Alternation with minimum delay. One cat failed to reach criterion performance on alternation after 150 days, i.e., after approximately 7,500 trials. At the end of this time he was responding rapidly but randomly to the two blocks. He was discontinued at this time.

One rabbit died during this phase of the training. The results for the remaining six cats and six rabbits are presented in Table 1.

There is no significant difference between cat and rabbit performance in the learning of the alternation task.

Delayed alternation. The remaining six cats achieved criterion performance for each of the four delay periods. of the remaining six rabbits one failed to achieve criterion on the $10 \mathrm{mec}$. delay task after 70 days and was discontinued at this stage. Results for the remaining six cats and five rabbits are presented in Table 2.

The Mann-Whitney $U$ test was used to test the significance of the difference in total number of days in

Table 2. Alternation with delay, number of days to criterion* for cats and rabbits

\begin{tabular}{lrrrrrrr} 
Cot no. & 2 & 3 & 4 & 5 & 6 & 7 & $\bar{X}$ \\
\hline 10 sec. & 6 & 18 & 7 & 4 & 25 & 7 & 11.17 \\
15 sec. & 4 & 6 & 13 & 6 & 4 & 4 & 6.17 \\
20 sec. & 4 & 9 & 8 & 9 & 10 & 6 & 7.67 \\
30 sec. & 8 & 4 & 11 & 6 & 14 & 5 & 8.00 \\
\hline Total & 22 & 37 & 39 & 25 & 53 & 22 & 33.00 \\
\hline Rabbit no. & $1(0)$ & $2(6)$ & $3(0)$ & $4(0)$ & $6(0)$ & $7(0)$ & $\bar{X}$ \\
\hline 10 sec. & 4 & 4 & 10 & 13 & 37 & $(a)$ & 13.60 \\
15 sec. & 16 & 5 & 25 & 5 & 27 & & 15.60 \\
20 sec. & 28 & 64 & 16 & 25 & 5 & & 27.60 \\
30 sec. & 7 & 13 & 11 & 8 & 13 & & 10.40 \\
\hline Total & 55 & 86 & 62 & 51 & 82 & & 67.20 \\
\hline
\end{tabular}

- This includes the 4 days during which criterion performance was being achieved.

(a) Failed to reach criterion on 10-sec. delay. training on delayed alternation between cats and rabbits. This difference is significant $(p<.01)$.

\section{Discussion}

While there is no significant difference between the rabbits and the cats in learning the alternation task with minimum delay, a quite striking and significant difference in level of performance becomes evident with the introduction of delay time, the cats mastering the delay task much more rapidly than the rabbits. The differences are most noticeable in the 15-sec. and 20sec. delay periods. This result is in the aniticipated direction in view of the higher cephalic index of brain body ratio for the cat.

With the use of increasing delay periods, rather than with random presentation of delays of differentlengths, it is apparent that both species could have extended beyond the 30-sec. delay period. Fletcher has discussed the significance of the use of these different approaches (Fletcher, 1965, pp. 148-149).

\section{References}

Fletcher, H. J. The delayed response problem. In A. M. Schrier, H. F. Harlow and F. Stollnitz (Ed.), Behavior of nonhuman primates. Vol. 1. New York: Academic Press, 1965. Pp. 129-165.

Hunter, W. S. The delayed reaction in a child. Psychol. Rev., $1917,24,74-87$.

Jacobsen, C. F., \& Nissen, H. W. Studies of cerebral function in primates: IV. The effects of frontal lobe lesions on the delayed alternation habit in monkeys. $J$. comp. psychol., 1937, 23, 101112.

Livesey, P. J. A note on double alternation by rabbits. J. comp. physiol. Psychol., 1964, 57, 104-107.

Livesey, P. J. Comparisons of double alternation performance of white rats, rabbits and cats. J. comp. physiol. Psychol., 1965, $59,155-158$.

Livesey, P. J. The rat, rabbit and cat in the Hebb-Williams Closed Field Test. Aust. J. Psychol., in press.

Miles, R. C., \& Blomquist, A. J. Frontal lesions and behavioral deficits in monkeys. $J$. Neurophysiol., 1960, 23, 471-484.

Mishkin, M., \& Pribram, K. H. Analysis of the effects of frontal lesions in monkeys: II. Variation of delayed response. $J$. con physiol. Psychol., 1956, 49, 39-40.

Pribram, K. H., Mishkin, M., Rosvold, H. E., \& Kaplan, S. J. Effects on delayed response performance of lesions of dorsolateral and ventromedial frontal cortex of baboons. J. comp. physiol Psychol., 1954, 45, 565-575.

Von Bonin, G. Brain weight and body weight of mammals. J. gen. Psychol., 1937, 16, 379-389.

Warden, C. J. Animal intelligence. Scient. American, 1951, 184.

Warren, J. M. Primate learning in comparative perspective. In A. M. Schrier, H. F. Harlow and F. Stollnitz (Eds.), Behavior of nonhuman primates, Vol. 1. New York: Academic Press, 1963. Pp. 249-281.

Note

1. This project was supported by a research grant from the University of Western Australia. 